\title{
Nutritional support and dietary interventions for patients with ulcerative colitis: current insights
}

\author{
This article was published in the following Dove Press journal: \\ Nutrition and Dietary Supplements \\ I5 April 2016 \\ Number of times this article has been viewed
}

\section{Rebecca J Hill \\ Children's Nutrition Research Centre, Child Health Research Centre, The University of Queensland, South Brisbane, Australia}

\begin{abstract}
Ulcerative colitis (UC) demonstrates a remitting and relapsing course, and patients have long believed diet plays a role in their symptoms. Ad hoc removal of foods and food groups from the diet without strong evidence for therapeutic benefit places patients at risk for nutritional deficiencies. This review discusses the need for nutritional support in UC and the role of dietary modification in its management in humans. Current evidence suggests patients with UC are not nutritionally compromised during remission, but with increasing disease activity, nutritional status is worth monitoring, in particular through body composition assessment and investigation for anemia. There is no clear evidence for dietary modulation to relieve symptoms in UC. Neither enteral nutrition nor parenteral nutrition is efficacious for symptom control or mucosal healing. While early studies suggested avoidance of dairy foods, no recent work has replicated these results. A low intake of insoluble fiber is recommended during acute disease flares; however, the role of fiber in modulating the gut microbiota and their metabolites warrants further attention. Several studies have investigated polyunsaturated fatty acids for UC; however, current evidence is not supportive for either inactive or active disease. There is emerging evidence that curcumin supplementation may be a new dietary treatment option. Often, evidence for therapeutic diets is difficult to interpret due to the reporting of combined results for both Crohn's disease and UC. In general, there is no evidenced specific dietary advice for patients with UC other than to follow healthy eating guidelines. Further work should determine if diet as treatment efficacy lies in modification of dietary patterns, thereby investigating the synergistic relationship between foods and their components, rather than investigation of single foods/nutrients. Large randomized controlled trials investigating dietary modification for UC are needed.
\end{abstract}

Keywords: nutritional status, malnutrition, diet, disease activity, body composition

\section{Introduction}

The role of nutrition, both as a therapy and a factor influencing etiology, is of interest for ulcerative colitis (UC). UC is thought to develop as a result of interactions between host genetics, the environment, immune reactivity, gut barrier functions, and alterations in the gut microbiome, ${ }^{1,2}$ all of which may be influenced by habitual diet through epigenetics and modulation of the resident microbiota. This has led researchers to attempt to link specific dietary components with the etiology of $\mathrm{UC}$, and further, to investigate dietary modification as a management strategy.

Patients with UC have long reported eating certain foods triggers symptoms. Food intolerance was self-reported by $64 \%$ of the patients with UC surveyed by Ballegaard et al; ${ }^{3}$ in the study of Jowett et al, ${ }^{4} 49 \%$ of patients with UC reported they
Correspondence: Rebecca J Hill

Children's Nutrition Research Centre,

Child Health Research Centre,

The University of Queensland, Level 6,

62 Graham Street, South Brisbane, QLD

$4 \mathrm{IOI}$, Australia

Tel +6I 730697232

Email r.hill@uq.edu.au
Nutrition and Dietary Supplements 2016:8 4I-49

(c) (1) () ๑ 2016 Hill. This work is published and licensed by Dove Medical Press Limited. The full terms of this license are avalable at https://www.dovepress.com/terms.php and (c) hereby accept the Terms. Non-commercial uses of the work are permitted without any further permision from Dove Medical Press Limited, provided the work is properly atribibued. For permission

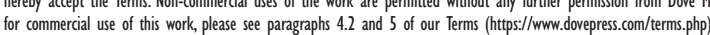

Dovepress

http://dx.doi.org/10.2147/NDS.S68/26

(1)


avoided certain foods; and Green et $\mathrm{al}^{5}$ reported $71 \%$ of pediatric patients with UC altered their diet following diagnosis.

Any elimination of particular foods or major food groups should be supported by clear evidence of intolerance, allergy, or therapeutic benefit because such restrictions influence the potential for nutrient deficiencies and malnutrition. Following any dietary restriction has risks, and there is evidence that food elimination diets can result in weight loss, failure to thrive, food aversions, eating disorders, and an increased risk of nutritional deficiency, especially in those who adhere long term to strict dietary eliminations. ${ }^{6,7}$ This is concerning for patients with UC where the potential for nutritional deficiencies already exists due to the nature of the disease itself.

Even with patient reports of a symptom-food relationship, randomized controlled trials are still lacking for the dietary management of UC. Further, management for UC should focus on both symptomatic control and achievement of mucosal healing. ${ }^{8}$ This review discusses the need for nutritional support in UC and the role of dietary modification in its management. It also, where possible, focuses on studies where results for patients with UC have been reported independently from those with Crohn's disease and studies conducted in humans, as evidence from experimental animal studies does not always translate to patient outcomes. This review does not discuss epidemiological studies for providing evidence of a dietary cause of UC. Gentschew and Ferguson have reviewed this area. ${ }^{1}$

\section{Are patients with UC nutritionally compromised? Nutritional status}

Although weight loss is associated with acute exacerbations of UC, the majority of evidence within the literature does not support malnutrition in these patients..$^{9-12}$ However, how malnutrition is assessed may play a role in these findings. With respect to the assessment of body composition as an indicator of nutritional status, Valentini et a ${ }^{13}$ reported significantly decreased lean body mass and body cell mass (BCM), assessed via bioelectrical impedance, in apparently well-nourished patients with UC compared with controls. Further, fat mass was significantly increased, and body mass index (BMI) was not different between patients and controls. These changes, suggestive of malnutrition, were not related to disease location, duration, or activity. More recently, utilizing dual-energy X-ray absorptiometry, Bryant et a ${ }^{14}$ described appendicular skeletal muscle (ASM) mass, as a reflection of true muscles mass in adult patients with UC. Of their cohort of 42 patients, $26 \%$ demonstrated an ASM index $<1$ standard deviation and were considered to have low lean mass; however, fat mass was not considered reduced. Our previous work has shown there are body compartment changes in children with UC, with loss of functional tissue (total body $\mathrm{K}^{40}$ measurement of $\mathrm{BCM}$ ) and an increase in fat mass. ${ }^{15}$ In our pediatric cohort, $20 \%$ of patients were considered malnourished with a BCM $z$-score less than the fifth percentile. In addition, there was a significant negative correlation with disease activity suggesting, as expected, a worsening of nutritional status with increased disease activity. As with the study of Valentini et al, ${ }^{13}$ standard measures of malnutrition, that is BMI, did not adequately classify patients who were malnourished when compared with measured body composition. While BMI is frequently used both clinically and in research as an indicator of nutritional status because of its ease of calculation, it is a proxy measure at best. BMI is a means of adjusting weight for height, and as such, gives information about a patient's body size. Although it can give an indication if the patients are overly large or small for their height, it does not provide information on body composition, and it is body composition that is a much more accurate measure of nutritional status. ${ }^{15}$

Other methods, such as serum albumin, are also often used to indicate malnutrition. However, albumin has been criticized for not being a valid marker of nutritional status but rather a marker of illness ${ }^{16}$ that is influenced by the inflammatory response irrespective of malnutrition. ${ }^{17}$ Data from our research group reported a significant positive relationship between BCM and albumin in patients with $\mathrm{UC}$, with serum albumin increasing with improving nutritional status, ${ }^{15}$ and as aforementioned, an inverse relationship was found between BCM and disease activity. However, further analysis of these data show albumin was also negatively correlated with disease activity $(r=-0.48, P=0.00)$ (unpublished data). These relationships support this criticism of using albumin as a marker of nutritional status, as any relationship between serum albumin and nutritional status may simply be a reflection of more severe inflammatory illness, rather than an indicator of nutritional compromise. Interestingly, Bryant et $\mathrm{al}^{14}$ found low serum albumin $(<34 \mathrm{~g} / \mathrm{dL})$ in only $7 \%$ of their cohort with UC, whereas $26 \%$ were found to have reduced functional lean mass according to the ASM index. Sixty-four percent of their patients had active disease. In contrast, Mijac et a ${ }^{18}$ concluded serum albumin has predictive value in classifying malnutrition in patients with active inflammatory bowel disease (IBD) (UC and Crohn's disease combined). They classified patients and controls according to unintentional weight loss, and anthropometric 
and biochemical measures, and while the combined group of patients with IBD were statistically different from control participants, the different methods employed for classification of malnutrition produced different proportions of patients as being malnourished (it was not reported whether these differences between methods were tested for significance). The difficulties with this study are the lack of a gold standard against which these measures can be compared, such as body composition assessment, and reported data for UC combined with Crohn's disease. While all patients had active disease, this ranged from mild to severe, and relationships between albumin and disease activity were not reported; that is, it is unknown if those patients classified as well nourished according to serum albumin were those with milder disease, or alternatively, those who were severely malnourished also had severely active disease, or whether disease activity categories were equally distributed across categories of malnutrition. In the study of Ripoli et al, ${ }^{19}$ no differences in serum albumin between active disease and remission states were reported. These studies highlight the difficultly of using serum albumin as a marker of nutritional status as it may be mediated by the inflammatory response rather than malnutrition.

\section{Micronutrient deficiencies}

With respect to specific nutrient deficiencies, anemia is reported as a problem for patients with UC. Gerasimidis et $\mathrm{al}^{20}$ described $79 \%$ of their cohort of newly diagnosed pediatric patients were either mildly or severely anemic, and that extensive colitis was associated with more severe anemia. Anemia persisted 1 year post-diagnosis for some patients, although the proportion of those considered as severe was reduced, with concurrent increases in the mild and non-anemia categories. The authors suggest this is indicative of the need for anemia-specific management for some patients with UC; however, their results showed neither blood transfusion nor oral iron supplementation was associated with improved anemia status at 1 year. A lower prevalence of anemia was described by Sjoberg et $\mathrm{al}^{21}$ in a population-based cohort (Uppsala Region, Sweden) of newly diagnosed patients with UC. In the group followed to 12 months post-diagnosis, baseline prevalence was $25.9 \%$ for moderate-to-severe anemia combined, reducing to $17.6 \%$ at 1 year follow-up. Pediatric patients with UC displayed significantly higher rates of anemia than adults at baseline, but at 1 year, rates were no longer different. Similar to the study of Gerasimidis et al, ${ }^{20}$ anemia was more prevalent at baseline in those with extensive disease. These patients displayed a significant reduction in anemia at 1 year.
Causes of anemia in patients with UC are the potentially reduced surface area in the bowel for nutrient absorption as a result of colitis or surgical removal, ${ }^{22}$ gastrointestinal blood losses, or a diet low in iron could be a contributing factor. ${ }^{20}$ Tsiountsioura et $\mathrm{al}^{9}$ found iron intakes below the lower level of recommended intakes in $18 \%$ of patients with $\mathrm{UC}$, and Ripoli et $\mathrm{al}^{19}$ reported significantly lower iron intake during periods of disease exacerbation. Although not statistically significant, there was a trend to reduced intake of meat during active disease, and this may have, in part, influenced the significant reduction in iron. Anemia is also associated with a folic acid deficit, and common treatment modalities for UC, such as methotrexate and sulfasalazine, can block the production and absorption of folate, respectively. ${ }^{22,23}$

There is limited evidence to suggest other micronutrient deficiencies are a problem for patients with UC. While vitamin D deficiency has been reported, the prevalence rate in patients with $\mathrm{UC}$ is similar to that seen in the general population (up to $45 \%{ }^{24,25}$ versus $30 \%-50 \%,{ }^{26}$ respectively), and therefore, does not appear to be a problem specific to UC. There are reports of low plasma concentrations of zinc in patients with $\mathrm{UC} ;{ }^{27,28}$ however, there are also reports to the contrary. ${ }^{29-31}$ Selenium may be a deficiency associated with UC; however, studies are limited..$^{27,31}$

\section{Summary on nutritional status}

There is limited evidence that nutritional support is necessary for patients with UC. For patients with active or extensive disease, anemia should be investigated and treated appropriately. Malnutrition should be assessed through measuring body composition, rather than anthropometry, in particular 1) in patients with ongoing active disease and 2) prior to surgery, as malnutrition is an independent risk factor for poor surgical outcomes. ${ }^{32}$

\section{Dietary management for UC Enteral and parenteral nutrition for UC}

Given the aforementioned lack of evidence for malnutrition in $\mathrm{UC}$, enteral feeding as a mode to rectify nutritional inadequacies is not supported. With respect to enteral and parenteral nutrition for achieving or maintaining remission in UC, the evidence is also unsupportive in this respect, and existing guidelines do not recommend their use in this manner. ${ }^{23,33}$ According to Wedrychowicz et al, ${ }^{34}$ a possible mechanism for the ineffectiveness of enteral nutrition has to do with modification of growth factor production, notably stimulation of transforming growth factor $\beta 1$ (TGF- $\beta 1$ ), which these authors reported occurs in patients with Crohn's disease in 
response to enteral nutrition but not those with UC. TGF- $\beta 1$ is evidenced to play a central role in the injury-repair process of the intestinal epithelium. ${ }^{34-37}$ Its expression is upregulated in the area of actively involved mucosa, with a higher concentration in the inflammatory cells closest to the mucosal surface indicating a role in epithelial cell restoration. ${ }^{35-37}$ Wedrychowicz et al, ${ }^{34}$ in their cohort of newly diagnosed pediatric patients naïve to pharmacological therapy, found patients with UC had increased serum TGF- $\beta 1$ compared with patients with Crohn's disease and control participants. While patients with Crohn's disease increased their serum TGF- $\beta 1$ during a 4-week course of enteral nutrition, patients with UC did not. Sambuelli et al, ${ }^{36}$ investigating patients with UC off treatment for at least 1 month, also found increased levels of serum TGF- $\beta 1$ at baseline compared with controls. After 1 week of steroid or sulfasalazine treatment, serum levels of TGF- $\beta 1$ increased; however, this increase was only significant in those patients considered as responders to therapy. By 42 days of treatment, levels of TGF- $\beta 1$ had significantly reduced in patients with UC compared with the day 7 measurement, and were no longer different to control values. In contrast, Sturm et $\mathrm{al}^{35}$ reported serum values of TGF- $\beta 1$ that did not differ between patients with UC and healthy controls. However, the patient characteristics in this study differ from those of Wedrychowicz et $\mathrm{al}^{34}$ and Sambuelli et $\mathrm{al},{ }^{36}$ in that Sturm et $\mathrm{a}^{35}$ did not assess patients pre- and posttreatment, but rather, measured TGF- $\beta 1$ in a cross-sectional sample of patients who would be assumed to be on ongoing therapy (although this is not specifically stated in the manuscript). While the disease activity of their patient group was spread over mild $(n=12)$, moderate $(n=8)$, and severe disease $(n=9)$, it may be that TGF- $\beta 1$ levels were reflective of ongoing therapy, and therefore, were consistent with normal values. With respect to mucosal sampling of TGF- $\beta 1$, Babyatsky et $\mathrm{al}^{37}$ reported increased levels of TGF- $\beta 1$ in the actively inflamed mucosa of both patients with UC and those with Crohn's disease, whereas minimal expression of TGF- $\beta 1$ was found in the uninflammed mucosa for both disease states.

It may be postulated from the aforementioned studies that serum TGF- $\beta 1$ levels may be associated with a therapeutic response to treatment via mucosal healing. As suggested by Wedrychowicz et al, ${ }^{34}$ it may be that enteral nutrition does not exert influence on TGF- $\beta 1$ for patients with UC, thereby limiting its effectiveness in this condition, whereas a factor associated with mucosal healing in patients with Crohn's disease following enteral nutrition is possibly modulation of this growth factor. Further evidence is still needed in this area to test these theories.

\section{Dairy}

In the 60 s, an early case series presented by Truelove, ${ }^{38}$ followed by a subsequent prospective controlled trial, ${ }^{39}$ suggested that a diet free of milk products maintained remission in UC, and upon reintroduction, caused relapse. Although the second study was a controlled trial, there was no randomization or blinding, and this early work has not been replicated through such a study design. Although the evidence for dairy exclusion is weak, patients with UC often report milk and dairy products cause problems and avoid its consumption, ${ }^{4}$ and clinicians have been reported to recommend restriction of milk product intake during periods of disease exacerbation. ${ }^{40}$ However, due to the absence of strong evidence supporting dairy product exclusion, general dietary guidelines by peak medical societies and IBD-related organizations do not recommend the elimination of dairy products, except where lactose intolerance is present. ${ }^{33}$ With respect to lactose intolerance in UC, Barrett et $\mathrm{al}^{41}$ reported a higher prevalence of lactose malabsorption in patients with UC compared with healthy controls; however, increasing age was associated with increased prevalence of lactose malabsorption. Participants of Chinese/South East Asian descent were excluded. In contrast, Mishkin et $\mathrm{al}^{42}$ reported a decreased prevalence of lactose malabsorption in patients with UC compared to controls, and both groups were considered at low ethnic risk for lactose malabsorption. Further, Bernstein et $\mathrm{a}^{40}$ concluded lactose intolerance was not related to the presence of UC but rather was more dependent on age and ethnicity. The limited research available does not support lactose intolerance being more prevalent in patients with UC compared with the general population.

\section{Fiber}

Along with milk and milk products, foods high in fiber, including fruits and vegetables, are commonly cited within the literature as being avoided/eliminated. ${ }^{4,43}$ General recommendations indicate to reduce the consumption of insoluble fiber (a low-residue diet), but this advice is associated with periods of increased disease activity and not recommended as necessary during remission. ${ }^{33,44,45}$

With respect to fiber consumption, there are concepts to consider: 1) fiber and its influence on motility and 2) fiber as a substrate for our resident gut microbiota. The construct around recommendations to reduce insoluble fiber is that this type of fiber contributes to stool bulk, increases bowel movements, and may raise the risk of obstruction. ${ }^{44}$ However, in cases of proctitis, where constipation is more of a problem, the World Gastroenterology Organisation 
suggests a high-residue diet may be beneficial. ${ }^{33}$ As opposed to insoluble fiber, soluble fibers are so named because they are water soluble. They add bulk to loose stools and can improve diarrhea by slowing transit time. ${ }^{44}$ In addition, soluble fiber can be readily fermented by the gut microbiota, producing by-products including short-chain fatty acids, and there is evidence that such by-products can be beneficial for UC, as described in the following text.

In particular, the metabolite butyrate is a fuel for colonic epithelial cells, ${ }^{46}$ enhances repair of injured gut epithelium, ${ }^{47}$ and exerts anti-inflammatory effects on the synthesis of inflammatory cytokines through the intracellular transcriptional factor nuclear factor kappa beta pathway. ${ }^{48}$ In theory, dietary substrates enhancing the production of butyrate would be of benefit to patients with UC. However, a reduced diversity of microbiota and lower abundance of butyrate-producing bacteria are associated with UC, ${ }^{49,50}$ and thus may influence the effectiveness of fiber as a therapy. ${ }^{51}$ This is supported by recent work by James et $\mathrm{al}^{51}$ where nonstarch polysaccharide and starch fermentation was found to be reduced in patients with UC, and increasing dietaryresistant starch and wheat bran in an 8-week, randomized cross-over trial did not correct the fermentative deficiency, even though there was a positive influence on gut motility. Aside from resistant starch and wheat bran, other dietary manipulations of fiber in UC have involved psyllium, ${ }^{52,53}$ germinated barley, ${ }^{54,55}$ inulin, ${ }^{56-58}$ and oat bran; ${ }^{59}$ however, the evidence for fiber in the management of $\mathrm{UC}$ is weak. A recent systematic review of randomized controlled trials ${ }^{60}$ only found positive effects for clinical end points or physiological outcomes in three of ten ${ }^{52,55,61}$ studies in UC and one of one ${ }^{62}$ in pouchitis.

Further avenues for modifying bacterial metabolites are to modulate the gut microbiota themselves through probiotic administration. As probiotics are live organisms, rather than foodstuffs or nutrients, their discussion is not within the scope of this review. The study by Orel and Kamhi Trop provides recent evidence on using probiotics as therapy in IBD. ${ }^{47}$

\section{Fatty acids}

The consumption of a western diet, with an increased intake of fat and fast foods, has long been associated with the etiology of IBD, and a recent systematic review of dietary intake and the risk of IBD has outlined several studies that report a link between high total fat intake, polyunsaturated fats, and omega-6 fatty acids in $\mathrm{UC},{ }^{63}$ particularly linoleic acid. ${ }^{64}$ With increased intakes of omega- 6 fatty acids, there is generally a reduction in the intake of omega-3 fatty acids, and it is potentially alteration in this ratio that is linked with IBD development. ${ }^{65}$

Omega-3 polyunsaturated fatty acids are derived from fish oil, with eicosapentaenoic acid and docosahexaenoic acid (DHA) being the two major fatty acid components. ${ }^{66}$ Both eicosapentaenoic acid and docosahexaenoic acid are anti-inflammatory in nature, and high dietary intakes of these lipids are shown to be associated with a low incidence of chronic inflammatory conditions. ${ }^{67}$ Metabolites of these fatty acids, prostaglandin $\mathrm{E}_{3}$ and leukotriene $\mathrm{B}_{5}$, also exert anti-inflammatory actions, and herein lies the rationale for their role in treatment of IBD.

Omega-3 fatty acids compete with arachidonic acid in the cyclooxygenase and lipoxygenase pathways. When arachidonic acid is the substrate for these pathways, the proinflammatory metabolites, prostaglandin $\mathrm{E}_{2}\left(\mathrm{PGE}_{2}\right)$ and leukotriene $\mathrm{B}_{4}\left(\mathrm{LTB}_{4}\right)$, are produced. $\mathrm{PGE}_{2}$ is known to increase vascular permeability and downregulate immune function ${ }^{68}$ and is a precursor to thromboxane $\mathrm{A}_{2}$. $\mathrm{LTB}_{4}$, formed primarily by inflammatory cells, is a potent chemotactic agent that is responsible for recruiting circulating neutrophils into inflamed tissue. ${ }^{69}$ Both $\mathrm{PGE}_{2}$ and $\mathrm{LTB}_{4}$ are markedly elevated in $\mathrm{UC},{ }^{70}$ and increased prostaglandin and thromboxane generation has been reported in Crohn's disease. ${ }^{71}$ Therefore, omega-3 polyunsaturated fatty acids competitively inhibit the formation of pro-inflammatory compounds and can result in the production of biological agents with anti-inflammatory effects. Further, production of inflammatory cytokines, namely interleukin-1 and tumor necrosis factor, have been shown to be decreased with omega-3 polyunsaturated fatty acid supplementation. ${ }^{72}$

The competitive characteristics of omega- 3 versus omega- 6 fatty acids underlie the rationale for nutritional therapies involving omega-3 polyunsaturated fatty acids. However, while this works in theory, it does not seem to translate into outcomes in intervention trials. A recent systematic review ${ }^{73}$ has highlighted many studies exhibit negative results in patients with UC, and further indicates many of the studies have methodological flaws. For example, small patient numbers and cross-over study designs are problematic for drawing firm conclusions on the usefulness of omega-3 fatty acids as a treatment for UC. Future studies need to consider the following: correct therapeutic dose; delivery method, that is, enteric-coated capsules; and appropriate placebo with inert characteristics. ${ }^{73}$

With respect to foods containing fatty acids, as opposed to supplementation as isolated nutrients, a small pilot study of 12 patients with mild UC investigated the effects 
of a diet of $600 \mathrm{~g}$ Atlantic salmon per week, in addition to continuation of current medication regiment. ${ }^{74} \mathrm{~A}$ significant reduction in the simple clinical colitis activity index was reported; however, this did not translate to changes in histology score. This study cohort was small, and there was a relatively high dropout rate of $26 \%$, with reasons for attrition associated with difficulty following the diet. Current evidence for fatty acid supplementation in UC is, therefore, not supported in either inactive or active disease.

\section{Curcumin}

In the past decade, there has been emerging evidence that curcumin has therapeutic benefits for patients with UC. ${ }^{75-78}$ Curcumin, which is present in turmeric, is a biologically active phytochemical with anti-inflammatory, antioxidant, and antitumor effects. ${ }^{79}$ Holt et al ${ }^{78}$ undertook a pilot study in five patients with ulcerative proctitis or proctosigmoiditis where they were administered $550 \mathrm{mg}$ of curcumin twice daily for 1 month, followed by $550 \mathrm{mg}$ three times a day for a further month. All patients showed improvement following the 2-month intervention, particularly for stool quality and frequency. Further, the need for medication was reduced, and serologic markers of inflammation returned to within normal limits. In a larger randomized, double-blind placebo-controlled trial of $2 \mathrm{~g}$ curcumin daily with concurrent sulfasalazine or mesalamine, Hanai et $\mathrm{al}^{17}$ reported significantly lower relapse rates in patients with quiescent $\mathrm{UC}$ on the active agent compared to the placebo $(4.6 \%$ versus $20.5 \%$, respectively). At the end of the 6-month intervention, the curcumin group significantly improved disease activity scores, as opposed to the placebo group whose disease activity score significantly worsened. While there was no change in the endoscopic index for the placebo group, there was significant improvement witnessed in the curcumin group. In the small tolerability study of Suskind et al, ${ }^{75}$ increasing doses of curcumin were tested in five patients with UC (remission or mild disease). Additional to standard therapy, patients were given $500 \mathrm{mg}$ of curcumin twice daily for 3 weeks, stepped up to $1 \mathrm{~g}$ twice daily for a further 3 weeks, and finally $2 \mathrm{~g}$ twice daily for the remaining 3 weeks. Although only three patients with UC completed the study (withdrawals were unrelated to the intervention), the two with mild disease decreased disease activity to values indicative of remission, and remission was maintained in the remaining one patient. Also, curcumin was shown to have benefit in a case study of an elderly woman with longstanding active UC. ${ }^{76}$ The patient was treated with $40 \mathrm{mg}$ per day prednisone in conjunction with $500 \mathrm{mg}$ curcumin, and after
1 month, bowel motions had decreased and steroid therapy had ceased. She remained in clinical remission for 12 months, and a colonoscopy at 10 months displayed findings consistent with chronic inactive UC.

Although the mechanism of action of curcumin is not fully defined, ${ }^{76}$ it is suggested to exert inhibitory influence on nuclear factor kappa beta and inducible nitric oxide synthase, ${ }^{79,80}$ thereby modulating the inflammatory cascade. The studies detailed show promise for curcumin as a dietary therapy for patients with UC, and curcumin was reported to be well tolerated in the studies. Further well-designed randomized double-blind placebo-controlled trials are needed.

\section{Other dietary components}

Both Magee et al ${ }^{81}$ and Jowett et $\mathrm{a}^{82}$ have investigated factors associated with relapse in UC. A high intake of meat, particularly red and processed meat, was shown to increase the odds of relapse. Further, relapse was also linked with high sulfur or sulfate intakes, and this may have in turn been influenced by meat intake, as processed meat in particular contains high levels of these constituents. ${ }^{82}$ Foods containing sulfite were indicated as detrimental for disease activity. ${ }^{81}$ In a small pilot study, Roediger ${ }^{83}$ reported no relapses or nutritional compromise over a combined observation period of 56 months, and a reduction in bowel motions per day, in four patients excluding foods containing high amounts of sulfur amino acids. However, the elimination diet was quite extensive (avoidance of eggs, cheese, full-cream milk, ice cream, mayonnaise, soy milk, mineral water, sulfited drinks, nuts, and cruciferous vegetables, with low intake of red meat), and it cannot be ruled out that other properties of some of these foods (eg, fermentable carbohydrates) may have been responsible for results found. There are no other large intervention studies investigating the effects of eliminating meat, meat products, or sulfite-containing foods on disease course in UC.

Evidence for other therapeutic diets is difficult to interpret due to the reporting of combined results for both Crohn's disease and UC. Herfarth et a ${ }^{84}$ reported improvement for a gluten-free diet in IBD. However, these results are confounded as the removal of gluten from the diet also constitutes a reduction in dietary fermentable oligo-, di-, and monosaccharides and polyols (FODMAPs). Gibson and Shepherd ${ }^{85}$ have suggested a mechanistic hypothesis for the link between FODMAPs and the etiology of Crohn's disease, and there is potentially a role for the manipulation of FODMAP intake for both Crohn's disease and UC due to these fermentable carbohydrates (which include fiber) being 
substrates for gut microbes, and hence, modulating their abundance and diversity within the gut. Gearry et $\mathrm{al}^{86}$ found 20 patients with UC had significant improvements in overall symptoms, abdominal pain, diarrhea, bloating, and wind after 3-6 months of following a low-FODMAP diet. Similar results were found for the patients with Crohn's disease.

One study has investigated the effect of comprehensive dietary guidelines focusing on multiple dietary components in a randomized controlled trial in UC. ${ }^{87}$ Patients were followed for 24 weeks and advised to follow the dietary guidelines for 4-6 weeks during a flare. These guidelines were to consume the following: 1) a low-fat diet, particularly saturated and trans fats; 2) a low-carbohydrate diet, avoiding refined simple carbohydrates and excess FODMAPs; 3) a low-fiber diet, particularly insoluble; 4) a high-protein diet but avoiding processed meats and reducing red meat intake; and 5) a diet involving probiotics. This advice essentially addressed factors associated with a typical "western" diet. A statistically significant reduction in the simple clinical colitis activity index was reported for the intervention group at 24 weeks; however, whether this was clinically relevant is not clear as the reduction failed to reach the value that was considered the minimum for clinical improvement. Although preliminary, this report provides evidence for considering dietary pattern and overall composition, rather than single nutrients or single foods/groups.

\section{Summary}

The evidence for diet modification as a therapy for UC is limited and is often hampered by reporting of results for both UC and Crohn's disease as one cohort. Certainly, the differences in gastrointestinal involvement between Crohn's disease and UC, and disparity in the efficacy of dietary treatments such as enteral nutrition, preclude extrapolation of results from studies in Crohn's disease to UC. Consideration needs to be given regarding whether certain dietary components in combination, that is, multiple properties within one or a combination of foods, will be more beneficial or if single-nutrient interventions should be the focus. Large randomized controlled trials of dietary manipulation are needed in this area.

\section{Conclusion}

While patients continue to report food influences symptoms in their UC, the evidence for a therapeutic diet remains elusive. Further, even if diet is able to control or minimize symptoms, there is no evidence that it is related to mucosal healing. Neither enteral nor parenteral nutrition, and associated bowel rest, is indicated for initiating or maintaining remission in UC. While patients with UC are not generally at risk of malnutrition, elimination of certain foods or whole food groups, without strong evidence for modulation of disease and monitoring by health professionals, places patients at risk for nutritional deficiencies.

\section{Disclosure}

$\mathrm{R}$ Hill is supported by Danone Pty Ltd for 2 days per week while working on a clinical trial unrelated to this review. The author reports no other conflicts of interest in this work.

\section{References}

1. Gentschew L, Ferguson LR. Role of nutrition and microbiota in susceptibility to inflammatory bowel diseases. Mol Nutr Food Res. 2012;56(4):524-535.

2. Lukas M, Bortlik M, Maratka Z. What is the origin of ulcerative colitis? Still more questions than answers. Postgrad Med J. 2006; 82(972):620-625.

3. Ballegaard M, Bjergstrom A, Brondum S, Hylander E, Jensen L, Ladefoged K. Self-reported food intolerance in chronic inflammatory bowel disease. Scand J Gastroenterol. 1997;32(6):569-571.

4. Jowett SL, Seal CJ, Phillips E, Gregory W, Barton JR, Welfare MR. Dietary beliefs of people with ulcerative colitis and their effect on relapse and nutrient intake. Clin Nutr. 2004;23(2):161-170.

5. Green TJ, Issenman RM, Jacobson K. Patients' diets and preferences in a pediatric population with inflammatory bowel disease. Can J Gastroenterol. 1998;12(8):544-549.

6. Gray P, Mehr S, Katelaris C, et al. Salicylate elimination diets in children: is food restriction supported by the evidence? Med J Aust. 2013;198(11):600-602.

7. Monsbakken KW, Vandvik PO, Farup PG. Perceived food intolerance in subjects with irritable bowel syndrome-etiology, prevalence and consequences. Eur J Clin Nutr. 2006;60(5):667-672.

8. Aloi M, Nuti F, Stronati L, Cucchiara S. Advances in the medical management of paediatric IBD. Nat Rev Gastroenterol Hepatol. 2014;11(2):99-108.

9. Tsiountsioura M, Wong JE, Upton J, et al. Detailed assessment of nutritional status and eating patterns in children with gastrointestinal diseases attending an outpatients clinic and contemporary healthy controls. Eur J Clin Nutr. 2014;68(6):700-706.

10. Wiskin AE, Wootton SA, Hunt TM, et al. Body composition in childhood inflammatory bowel disease. Clin Nutr. 2011;30(1):112-115.

11. Tanaka M, Shibayama T, Sato E, Sasaki S, Kazuma K. Dietary intake and body composition of Japanese ulcerative colitis patients in remission. Gastroenterol Nurs. 2007;30(5):357-362.

12. Capristo E, Mingrone G, Addolorato G, Greco AV, Gasbarrini G. Metabolic features of inflammatory bowel disease in a remission phase of the disease activity. J Intern Med. 1998;243(5):339-347.

13. Valentini L, Schaper L, Buning C, et al. Malnutrition and impaired muscle strength in patients with Crohn's disease and ulcerative colitis in remission. Nutrition. 2008;24(7-8):694-702.

14. Bryant RV, Ooi S, Schultz CG, et al. Low muscle mass and sarcopenia: common and predictive of osteopenia in inflammatory bowel disease. Aliment Pharmacol Ther. 2015;41(9):895-906.

15. Hill RJ, Davies PS. You look all right to me: compromised nutritional status in paediatric patients with ulcerative colitis. $J$ Pediatr Gastroenterol Nutr. 2013;56(4):385-389.

16. Friedman AN, Fadem SZ. Reassessment of albumin as a nutritional marker in kidney disease. J Am Soc Nephrol. 2010;21:223-230.

17. Valentini L, Schulzke JD. Mundane, yet challenging: the assessment of malnutrition in inflammatory bowel disease. Eur J Intern Med. 2011;22(1):13-15. 
18. Mijac DD, Jankovic GL, Jorga J, Krstic MN. Nutritional status in patients with active inflammatory bowel disease: prevalence of malnutrition and methods for routine nutritional assessment. Eur J Intern Med. 2010;21(4):315-319.

19. Ripoli J, Miszputen SJ, Ambrogini Jr O, Carvalho L. Nutritional follow-up of patients with ulcerative colitis during periods of intestinal inflammatory activity and remission. Arq Gastroenterol. 2010;47(1):49-55.

20. Gerasimidis K, Barclay A, Papangelou A, et al. The epidemiology of anemia in pediatric inflammatory bowel disease: prevalence and associated factors at diagnosis and follow-up and the impact of exclusive enteral nutrition. Inflamm Bowel Dis. 2013;19(11):2411-2422.

21. Sjoberg D, Holmstrom T, Larsson M, Nielsen AL, Holmquist L, Ronnblom A. Anemia in a population-based IBD cohort (ICURE): still high prevalence after 1 year, especially among pediatric patients. Inflamm Bowel Dis. 2014;20(12):2266-2270.

22. Mullin GE. Micronutrients and inflammatory bowel disease. Nutr Clin Pract. 2012;27(1):136-137.

23. Lochs H, Dejong C, Hammarqvist F, et al. ESPEN Guidelines on Enteral Nutrition: Gastroenterology. Clin Nutr. 2006;25(2):260-274.

24. Blanck S, Aberra F. Vitamin d deficiency is associated with ulcerative colitis disease activity. Dig Dis Sci. 2013;58(6):1698-1702.

25. Pappa HM, Grand RJ, Gordon CM. Report on the vitamin D status of adult and pediatric patients with inflammatory bowel disease and its significance for bone health and disease. Inflamm Bowel Dis. 2006;12(12):1162-1174.

26. Lee JH, O'Keefe JH, Bell D, Hensrud DD, Holick MF. Vitamin D deficiency an important, common, and easily treatable cardiovascular risk factor? J Am Coll Cardiol. 2008;52(24):1949-1956.

27. Geerling BJ, Badart-Smook A, Stockbrügger RW, Brummer R-JM. Comprehensive nutritional status in recently diagnosed patients with inflammatory bowel disease compared with population controls. Eur J Clin Nutr. 2000;54:514-521.

28. Janczewska I, Bartnik W, Butruk E, Tomecki R, Kazik E, Ostrowski J. Metabolism of vitamin A in inflammatory bowel disease. Hepatogastroenterology. 1991;38(5):391-395.

29. Sikora SK, Spady D, Prosser C, El-Matary W. Trace elements and vitamins at diagnosis in pediatric-onset inflammatory bowel disease. Clin Pediatr. 2011;50(6):488-492.

30. Dalekos GN, Ringstad J, Savaidis I, Seferiadis KI, Tsianos EV. Zinc, copper and immunological markers in the circulation of well nourished patients with ulcerative colitis. Eur J Gastroenterol Hepatol. 1998;10(4):331-337.

31. Ringstad J, Kildebo S, Thomassen Y. Serum selenium, copper, and zinc concentrations in Crohn's disease and ulcerative colitis. Scand J Gastroenterol. 1993;28(7):605-608.

32. Markel TA, Lou DC, Pfefferkorn M, et al. Steroids and poor nutrition are associated with infectious wound complications in children undergoing first stage procedures for ulcerative colitis. Surgery. 2008;144(4):540-545.

33. Brown AC, Rampertab SD, Mullin GE. Existing dietary guidelines for Crohn's disease and ulcerative colitis. Expert Rev Gastroenterol Hepatol. 2011;5(3):411-425.

34. Wedrychowicz A, Kowalska-Duplaga K, Jedynak-Wasowicz U, et al. Serum concentrations of VEGF and TGF-beta1 during exclusive enteral nutrition in IBD. J Pediatr Gastroenterol Nutr. 2011;53(2):150-155.

35. Sturm A, Schulte C, Schatton R, et al. Transforming growth factor-beta and hepatocyte growth factor plasma levels in patients with inflammatory bowel disease. Eur J Gastroenterol Hepatol. 2000;12(4):445-450.

36. Sambuelli A, Diez RA, Sugai E, et al. Serum transforming growth factor-betal levels increase in response to successful antiinflammatory therapy in ulcerative colitis. Aliment Pharmacol Ther. 2000;14(11):1443-1449.

37. Babyatsky MW, Rossiter G, Podolsky DK. Expression of transforming growth factors alpha and beta in colonic mucosa in inflammatory bowel disease. Gastroenterology. 1996;110(4):975-984.

38. Truelove SC. Ulcerative colitis provoked by milk. BMJ. 1961;1(5220): 154-160.
39. Wright R, Truelove SC. A controlled therapeutic trial of various diets in ulcerative colitis. BMJ. 1965;2(5454):138-141.

40. Bernstein CN, Ament M, Artinian L, Ridgeway J, Shanahan F. Milk tolerance in adults with ulcerative colitis. Am J Gastroenterol. 1994;89(6):872-877.

41. Barrett JS, Irving PM, Shepherd SJ, Muir JG, Gibson PR. Comparison of the prevalence of fructose and lactose malabsorption across chronic intestinal disorders. Aliment Pharmacol Ther. 2009;30(2):165-174.

42. Mishkin B, Yalovsky M, Mishkin S. Increased prevalence of lactose malabsorption in Crohn's disease patients at low risk for lactose malabsorption based on ethnic origin. Am J Gastroenterol. 1997;92(7):1148-1153.

43. Walton M, Alaunyte I. Do patients living with ulcerative colitis adhere to healthy eating guidelines? A cross-sectional study. Br J Nutr. 2014;112(10):1628-1635.

44. Hwang C, Ross V, Mahadevan U. Popular exclusionary diets for inflammatory bowel disease: the search for a dietary culprit. Inflamm Bowel Dis. 2014;20(4):732-741.

45. Cabre E, Domenech E. Impact of environmental and dietary factors on the course of inflammatory bowel disease. World J Gastroenterol. 2012;18(29):3814-3822.

46. Roediger WE. Role of anaerobic bacteria in the metabolic welfare of the colonic mucosa in man. Gut. 1980;21(9):793-798.

47. Orel R, Kamhi Trop T. Intestinal microbiota, probiotics and prebiotics in inflammatory bowel disease. World $J$ Gastroenterol. 2014;20(33):11505-11524.

48. Kanauchi O, Matsumoto Y, Matsumura M, Fukuoka M, Bamba T. The beneficial effects of microflora, especially obligate anaerobes, and their products on the colonic environment in inflammatory bowel disease. Curr Pharm Des. 2005;11(8):1047-1053.

49. Benus RF, van der Werf TS, Welling GW, et al. Association between Faecalibacterium prausnitzii and dietary fibre in colonic fermentation in healthy human subjects. Br J Nutr. 2010;104(5):693-700.

50. Sokol H, Seksik P, Furet JP, et al. Low counts of Faecalibacterium prausnitzii in colitis microbiota. Inflamm Bowel Dis. 2009;15(8): 1183-1189.

51. James SL, Christophersen CT, Bird AR, et al. Abnormal fibre usage in UC in remission. Gut. 2015;64(4):562-570.

52. Fernandez-Banares F, Hinojosa J, Sanchez-Lombrana JL, et al. Randomized clinical trial of Plantago ovata seeds (dietary fiber) as compared with mesalamine in maintaining remission in ulcerative colitis. Spanish Group for the Study of Crohn's Disease and Ulcerative Colitis (GETECCU). Am J Gastroenterol. 1999;94(2):427-433.

53. Hallert C, Kaldma M, Petersson BG. Ispaghula husk may relieve gastrointestinal symptoms in ulcerative colitis in remission. Scand J Gastroenterol. 1991;26(7):747-750.

54. Faghfoori Z, Navai L, Shakerhosseini R, Somi MH, Nikniaz Z, Norouzi MF. Effects of an oral supplementation of germinated barley foodstuff on serum tumour necrosis factor-alpha, interleukin- 6 and -8 in patients with ulcerative colitis. Ann Clin Biochem. 2011;48(Pt 3): 233-237.

55. Kanauchi O, Suga T, Tochihara M, et al. Treatment of ulcerative colitis by feeding with germinated barley foodstuff: first report of a multicenter open control trial. J Gastroenterol. 2002;37 (Suppl)14:67-72.

56. Casellas F, Borruel N, Torrejon A, et al. Oral oligofructose-enriched inulin supplementation in acute ulcerative colitis is well tolerated and associated with lowered faecal calprotectin. Aliment Pharmacol Ther. 2007;25(9):1061-1067.

57. Federico A, Tuccillo C, Grossi E, et al. The effect of a new symbiotic formulation on plasma levels and peripheral blood mononuclear cell expression of some pro-inflammatory cytokines in patients with ulcerative colitis: a pilot study. Eur Rev Med Pharmacol Sci. 2009;13(4):285-293.

58. Furrie E, Macfarlane S, Kennedy A, et al. Synbiotic therapy (Bifidobacterium longum/Synergy 1) initiates resolution of inflammation in patients with active ulcerative colitis: a randomised controlled pilot trial. Gut. 2005;54(2):242-249.

59. Hallert C, Bjorck I, Nyman M, Pousette A, Granno C, Svensson H. Increasing fecal butyrate in ulcerative colitis patients by diet: controlled pilot study. Inflamm Bowel Dis. 2003;9(2):116-121. 
60. Wedlake L, Slack N, Andreyev HJ, Whelan K. Fiber in the treatment and maintenance of inflammatory bowel disease: a systematic review of randomized controlled trials. Inflamm Bowel Dis. 2014;20(3):576-586.

61. Copaci I, Chira C, Rovinaru I, Micu L. Maintenance of remission of ulcerative colitis (UC): mesalamine, dietary fiber, S. Boulardi. Dig Liver Dis. 2000;32(Suppl 1):A24.

62. Welters CF, Heineman E, Thunnissen FB, van den Bogaard AE, Soeters PB, Baeten CG. Effect of dietary inulin supplementation on inflammation of pouch mucosa in patients with an ileal pouch-anal anastomosis. Dis Colon Rectum. 2002;45(5):621-627.

63. Hou JK, Abraham B, El-Serag H. Dietary intake and risk of developing inflammatory bowel disease: a systematic review of the literature. $\mathrm{Am}$ J Gastroenterol. 2011;106(4):563-573.

64. Hart AR, Luben R, Olsen A, et al. Diet in the aetiology of ulcerative colitis: a European prospective cohort study. Digestion. 2008;77(1): 57-64.

65. Sung MK, Park MY. Nutritional modulators of ulcerative colitis: clinical efficacies and mechanistic view. World J Gastroenterol. 2013; 19(7):994-1004

66. Wardlaw GM, Insel PM. Perspectives in Nutrition. 3rd ed. St. Louis: Mosby-Year Book, Inc; 1996.

67. Kromann N, Green A. Epidemiological studies in the Upernavik district, Greenland: incidence of some chronic diseases. Acta Med Scand. 1980;208:401-406.

68. Davies P, Bailey PJ, Goldenberg M, Ford-Hutchinson AW. The role of arachidonic acid oxygenation products in pain and inflammation. Ann Rev Immunol. 1984;2:335-357.

69. Ford-Hutchinson AW, Bray MA, Doig MV, Shipley ME, Smith JH. Leukotriene B, a potent chemotactic and aggregating substance released from polymorphonuclear leukocytes. Nature. 1984;266:264-265.

70. Lauritsen K, Laursen LS, Bukhave K, Rask-Madsen J. Effects of topical 5-aminosalicylic acid and prednisolone on prostaglandin $\mathrm{E}_{2}$, and leukotriene $\mathrm{B}_{4}$ levels determined by equilibrium in vivo dialysis of rectum in relapsing ulcerative colitis. Gastroenterology. 1986;91: 837-844.

71. Lorenz R, Weber PC, Szimnau P, Heldwein W, Strasser T, Loeschke K. Supplementation with n-3 fatty acids from fish oil in chronic inflammatory bowel disease-a randomised, placebo-controlled, double-blind cross-over trial. J Intern Med. 1989;225(Suppl 1):225-232.

72. Endres S, Ghorbani R, Kelly VE, et al. The effect of dietary supplementation with $n-3$ fatty acids on the synthesis of interleukin-1 and tumor necrosis factor by mononuclear cells. New Eng J Med. 1989;320:265-270.

73. Cabre E, Manosa M, Gassull MA. Omega-3 fatty acids and inflammatory bowel diseases-a systematic review. Br J Nutr. 2012;107(Supp1 2): S240-S252.
74. Grimstad T, Berge RK, Bohov P, et al. Salmon diet in patients with active ulcerative colitis reduced the simple clinical colitis activity index and increased the anti-inflammatory fatty acid index--a pilot study. Scand J Clin Lab Invest. 2011;71(1):68-73.

75. Suskind DL, Wahbeh G, Burpee T, Cohen M, Christie D, Weber W. Tolerability of curcumin in pediatric inflammatory bowel disease: a forced-dose titration study. J Pediatr Gastroenterol Nutr. 2013;56(3): 277-279.

76. Lahiff C, Moss AC. Curcumin for clinical and endoscopic remission in ulcerative colitis. Inflamm Bowel Dis. 2011;17(7):E66.

77. Hanai H, Iida T, Takeuchi K, et al. Curcumin maintenance therapy for ulcerative colitis: randomized, multicenter, double-blind, placebocontrolled trial. Clin Gastroenterol Hepatol. 2006;4(12):1502-1506.

78. Holt PR, Katz S, Kirshoff R. Curcumin therapy in inflammatory bowel disease: a pilot study. Dig Dis Sci. 2005;50(11):2191-2193.

79. Ramsewak RS, DeWitt DL, Nair MG. Cytotoxicity, antioxidant and anti-inflammatory activities of curcumins I-III from Curcuma longa. Phytomedicine. 2000;7(4):303-308.

80. Venkataranganna MV, Rafiq M, Gopumadhavan S, Peer G, Babu UV, Mitra SK. NCB-02 (standardized Curcumin preparation) protects dinitrochlorobenzene-induced colitis through down-regulation of NFkappa-B and iNOS. World J Gastroenterol. 2007;13(7): 1103-1107.

81. Magee EA, Edmond LM, Tasker SM, Kong SC, Curno R, Cummings JH. Associations between diet and disease activity in ulcerative colitis patients using a novel method of data analysis. Nutr J. 2005;4:7.

82. Jowett SL, Seal CJ, Pearce MS, et al. Influence of dietary factors on the clinical course of ulcerative colitis: a prospective cohort study. Gut. 2004;53(10):1479-1484.

83. Roediger WE. Decreased sulphur aminoacid intake in ulcerative colitis Lancet. 1998;351(9115):1555.

84. Herfarth HH, Martin CF, Sandler RS, Kappelman MD, Long MD. Prevalence of a gluten-free diet and improvement of clinical symptoms in patients with inflammatory bowel diseases. Inflamm Bowel Dis 2014;20(7):1194-1197.

85. Gibson PR, Shepherd SJ. Personal view: food for thought-western lifestyle and susceptibility to Crohn's disease. The FODMAP hypothesis. Aliment Pharmacol Ther. 2005;21(12):1399-1409.

86. Gearry RB, Irving PM, Barrett JS, Nathan DM, Shepherd SJ, Gibson PR. Reduction of dietary poorly absorbed short-chain carbohydrates (FODMAPs) improves abdominal symptoms in patients with inflammatory bowel disease-a pilot study. J Crohns Colitis. 2009;3(1):8-14.

87. Kyaw MH, Moshkovska T, Mayberry J. A prospective, randomized, controlled, exploratory study of comprehensive dietary advice in ulcerative colitis: impact on disease activity and quality of life. Eur J Gastroenterol Hepatol. 2014;26(8):910-917.
Nutrition and Dietary Supplements

\section{Publish your work in this journal}

Nutrition and Dietary Supplements is an international, peer-reviewed, open access journal focusing on research into nutritional requirements in health and disease, impact on metabolism and the identification and optimal use of dietary strategies and supplements necessary for normal growth and development. The journal welcomes papers covering

\section{Dovepress}

original research, basic science, clinical \& epidemiological studies, reviews and evaluations, guidelines, expert opinion and commentary, case reports and extended reports. The manuscript management system is completely online and includes a very quick and fair peer-review system, which is all easy to use. 